\title{
Strong convergence theorem for amenable semigroups of nonexpansive mappings and variational inequalities
}

Hossein Piri ${ }^{*}$ and Ali Haji Badali

\author{
* Correspondence: $h$. \\ piri@bonabetu.ac.ir \\ Department of Mathematics, \\ University of Bonab, Bonab 55517- \\ 61167, Iran
}

\begin{abstract}
In this paper, using strongly monotone and lipschitzian operator, we introduce a general iterative process for finding a common fixed point of a semigroup of nonexpansive mappings, with respect to strongly left regular sequence of means defined on an appropriate space of bounded real-valued functions of the semigroups and the set of solutions of variational inequality for $\beta$-inverse strongly monotone mapping in a real Hilbert space. Under suitable conditions, we prove the strong convergence theorem for approximating a common element of the above two sets.

Mathematics Subject Classification 2000: 47H09, 47H10, 43A07, 47J25

Keywords: projection, common fixed point, amenable semigroup, iterative process, strong convergence, variational inequality
\end{abstract}

\section{Introduction}

Throughout this paper, we assume that $H$ is a real Hilbert space with inner product and norm are denoted by $\langle.$, . $\rangle$ and $\|$. $\|$, respectively, and let $C$ be a nonempty closed convex subset of $H$. A mapping $T$ of $C$ into itself is called nonexpansive if $\| T x-T y$ $\|\leq\| x-y \|$, for all $x, y \in H$. By Fix(T), we denote the set of fixed points of $T$ (i.e., Fix $(T)=\{x \in H: T x=x\})$, it is well known that $F i x(T)$ is closed and convex. Recall that a self-mapping $f: C \rightarrow C$ is a contraction on $C$ if there exists a constant $\alpha \in[0,1)$ such that $\|f(x)-f(y)\| \leq \alpha\|x-y\|$ for all $x, y \in C$.

Let $B: C \rightarrow H$ be a mapping. The variational inequality problem, denoted by $V I(C$, $B)$, is to fined $x \in C$ such that

$$
\langle B x, y-x\rangle \geq 0
$$

for all $y \in C$. The variational inequality problem has been extensively studied in literature. See, for example, $[1,2]$ and the references therein.

Definition 1.1 Let $B: C \rightarrow H$ be a mapping. Then $B$

(1) is called $\eta$-strongly monotone if there exists a positive constant $\eta$ such that

$$
\langle B x-B y, x-y\rangle \geq \eta\|x-y\|^{2}, \quad \forall x, y \in C,
$$


(2) is called $k$-Lipschitzian if there exist a positive constant $k$ such that

$$
\|B x--B y\| \leq k\|x-y\|, \quad \forall x, y \in C,
$$

(3) is called $\beta$-inverse strongly monotone if there exists a positive real number $\beta>0$ such that

$$
\langle B x-B y, x-y\rangle \geq \beta\|B x-B y\|^{2}, \quad \forall x, y \in C .
$$

It is obvious that any $\beta$-inverse strongly monotone mapping $B$ is $\frac{1}{\beta}$-Lipschitzian.

Moudafi [3] introduced the viscosity approximation method for fixed point of nonexpansive mappings (see [4] for further developments in both Hilbert and Banach spaces). Starting with an arbitrary initial $x_{0} \in H$, define a sequence $\left\{x_{n}\right\}$ recursively by

$$
x_{n+1}=\left(1-\alpha_{n}\right) T x_{n}+\alpha_{n} f\left(x_{n}\right), \quad n \geq 0,
$$

where $\alpha_{n}$ is sequence in $(0,1) . \mathrm{Xu}[4,5]$ proved that under certain appropriate conditions on $\left\{\alpha_{n}\right\}$, the sequences $\left\{x_{n}\right\}$ generated by (2) strongly converges to the unique solution $x^{*}$ in $\operatorname{Fix}(T)$ of the variational inequality:

$$
\left\langle(f-I) x^{*}, x-x^{*}\right\rangle \leq 0, \quad \forall x \in \operatorname{Fix}(T) .
$$

Let $A$ is strongly positive operator on $C$. That is, there is a constant $\bar{\gamma}>0$ with the property that

$$
\langle A x, x\rangle \geq \bar{\gamma}\|x\|^{2}, \quad \forall x \in C .
$$

In [5], it is proved that the sequence $\left\{x_{n}\right\}$ generated by the iterative method bellow with initial guess $x_{0} \in H$ chosen arbitrarily,

$$
x_{n+1}=\left(I-\alpha_{n} A\right) T x_{n}+\alpha_{n} u, \quad n \geq 0,
$$

converges strongly to the unique solution of the minimization problem

$$
\min _{x \in \operatorname{Fix}(T)} \frac{1}{2}\langle A x, x\rangle-\langle x, b\rangle,
$$

where $b$ is a given point in $H$.

Combining the iterative method (2) and (3), Marino and Xu [6] consider the following iterative method:

$$
x_{n+1}=\left(I-\alpha_{n} A\right) T x_{n}+\alpha_{n} \gamma f\left(x_{n}\right), \quad n \geq 0,
$$

it is proved that if the sequence $\left\{\alpha_{n}\right\}$ of parameters satisfies the following conditions:

$\left(C_{1}\right) \alpha_{n} \rightarrow 0$,

(C) $\sum_{n=0}^{\infty} \alpha_{n}=\infty$,

$C_{3}$ ) either $\sum_{n=0}^{\infty}\left|\alpha_{n+1}-\alpha_{n}\right|<\infty$ or $\lim _{n \rightarrow \infty} \frac{\alpha_{n+1}}{\alpha_{n}}=1$.

then, the sequence $\left\{x_{n}\right\}$ generated by (4) converges strongly, as $n \rightarrow \infty$, to the unique solution of the variational inequality:

$$
\left\langle(\gamma f-A) x^{*}, x-x^{*}\right\rangle \leq 0, \quad \forall x \in \operatorname{Fix}(T),
$$


which is the optimality condition for minimization problem

$$
\min _{x \in F i x(T)} \frac{1}{2}\langle A x, x\rangle-h(x)
$$

where $h$ is a potential function for $\gamma f$ (i.e., $h^{\prime}(x)=\gamma f(x)$, for all $x \in H$ ). Some people also study the application of the iterative method $(4)[7,8]$.

Yamada [9] introduce the following hybrid iterative method for solving the variational inequality:

$$
x_{n+1}=T x_{n}-\mu \alpha_{n} F\left(T x_{n}\right), \quad n \in \mathbb{N},
$$

where $F$ is $k$-Lipschitzian and $\eta$-strongly monotone operator with $k>0, \eta>0$, $0<\mu<\frac{2 \eta}{k^{2}}$, then he proved that if $\left\{\alpha_{n}\right\}$ satisfying appropriate conditions, then $\left\{x_{n}\right\}$ generated by (5) converges strongly to the unique solution of the variational inequality:

$$
\left\langle F x^{*}, x-x^{*}\right\rangle \geq 0, \quad \forall x \in \operatorname{Fix}(T) .
$$

In 2010, Tian [10] combined the iterative (4) with the iterative method (5) and considered the iterative methods:

$$
x_{n+1}=\left(I-\mu \alpha_{n} F\right) T x_{n}+\alpha_{n} \gamma f\left(x_{n}\right), \quad n \geq 0,
$$

and he prove that if the sequence $\left\{\alpha_{n}\right\}$ of parameters satisfies the conditions $\left(C_{1}\right)$, $\left(C_{2}\right)$, and $\left(C_{3}\right)$, then the sequences $\left\{x_{n}\right\}$ generated by (6) converges strongly to the unique solution $x^{*} \in \operatorname{Fix}(T)$ of the variational inequality:

$$
\left\langle(\mu F-\gamma f) x^{*}, x-x^{*}\right\rangle \geq 0, \quad \forall x \in \operatorname{Fix}(T) .
$$

In this paper motivated and inspired by Atsushiba and Takahashi [11], Ceng and Yao [12], Kim [13], Lau et al. [14], Lau et al [15], Marino and Xu [6], Piri and Vaezi [16], Tian [10], Xu [5] and Yamada [9], we introduce the following general iterative algorithm: Let $x_{0} \in C$ and

$$
\left\{\begin{array}{l}
y_{n}=\beta_{n} x_{n}+\left(1-\beta_{n}\right) P_{C}\left(x_{n}-\delta_{n} B x_{n}\right), \\
x_{n+1}=\alpha_{n} \gamma f\left(x_{n}\right)+\left(I-\alpha_{n} \mu F\right) T_{\mu_{n}} y_{n}, \quad n \geq 0 .
\end{array}\right.
$$

where $P_{C}$ is a metric projection of $\mathrm{H}$ onto $\mathrm{C}, \mathrm{B}$ is $\beta$-inverse strongly monotone, $\phi=$ $\left\{T_{t}: t \in S\right\}$ is a nonexpansive semigroup on $H$ such that the set $\mathcal{F}=\operatorname{Fix}(\varphi) \cap V I(C, B) \neq \emptyset_{n}, X$ is a subspace of $B(S)$ such that $1 \in X$ and the mapping $t$ $\rightarrow\left\langle T_{t} x, y\right\rangle$ is an element of $X$ for each $x, y \in H$, and $\left\{\mu_{n}\right\}$ is a sequence of means on $X$. Our purpose in this paper is to introduce this general iterative algorithm for approximating a common element of the set of common fixed point of a semigroup of nonexpansive mappings and the set of solutions of variational inequality for $\beta$-inverse strongly monotone mapping which solves some variational inequality. We will prove that if $\left\{\mu_{n}\right\}$ is left regular and the sequences $\left\{\alpha_{n}\right\},\left\{\beta_{n}\right\}$, and $\left\{\delta_{n}\right\}$ of parameters satisfies appropriate conditions, then the sequences $\left\{x_{n}\right\}$ and $\left\{y_{n}\right\}$ generated by (7) converges strongly to the unique solution $x^{*} \in \mathcal{F}$ of the variational inequalities:

$$
\begin{cases}\left\langle(\mu F-\gamma f) x^{*}, x-x^{*}\right\rangle \geq 0, & \forall x \in \mathcal{F} \\ \left\langle B x^{*}, y-x^{*}\right\rangle \geq 0 & \forall y \in C\end{cases}
$$




\section{Preliminaries}

Let $S$ be a semigroup and let $B(S)$ be the space of all bounded real-valued functions defined on $S$ with supremum norm. For $s \in S$ and $f \in B(S)$, we define elements $l_{s} f$ and $r_{s} f$ in $B(S)$ by

$$
\left(l_{s} f\right)(t)=f(s t), \quad\left(r_{s} f\right)(t)=f(t s), \quad \forall t \in S .
$$

Let $X$ be a subspace of $B(S)$ containing 1 and let $X^{*}$ be its topological dual. An element $\mu$ of $X^{*}$ is said to be a mean on $X$ if $\|\mu\|=\mu(1)=1$. We often write $\mu_{t}(f(t))$ instead of $\mu(f)$ for $\mu \in X^{*}$ and $f \in X$. Let $X$ be left invariant (resp. right invariant), i.e., $l_{s}(X) \subset X\left(\right.$ resp. $\left.r_{s}(X) \subset X\right)$ for each $s \in S$. A mean $\mu$ on $X$ is said to be left invariant (resp. right invariant) if $\mu\left(l_{s} f\right)=\mu(f)$ (resp. $\mu\left(r_{s} f\right)=\mu(f)$ ) for each $s \in S$ and $f \in X$. X is said to be left (resp. right) amenable if $X$ has a left (resp. right) invariant mean. $X$ is amenable if $X$ is both left and right amenable. As is well known, $B(S)$ is amenable when $S$ is a commutative semigroup, see [15]. A net $\left\{\mu_{\alpha}\right\}$ of means on $X$ is said to be strongly left regular if

$$
\lim _{\alpha}\left\|l_{s}^{*} \mu_{\alpha}-\mu_{\alpha}\right\|=0,
$$

for each $s \in S$, where $l_{s}^{*}$ is the adjoint operator of $l_{s}$.

Let $S$ be a semigroup and let $C$ be a nonempty closed and convex subset of a reflexive Banach space $E$. A family $\phi=\left\{T_{t}: t \in S\right\}$ of mapping from $C$ into itself is said to be a nonexpansive semigroup on $C$ if $T_{t}$ is nonexpansive and $T_{t s}=T_{t} T_{s}$ for each $t, s \in$ S. By Fix $(\phi)$, we denote the set of common fixed points of $\phi$, i.e.,

$$
\operatorname{Fix}(\varphi)=\bigcap_{t \in S}\left\{x \in C: T_{t} x=x\right\} .
$$

Lemma 2.1 [15] Let $S$ be a semigroup and $C$ be a nonempty closed convex subset of a reflexive Banach space $E$. Let $\phi=\left\{T_{t}: t \in S\right\}$ be a nonexpansive semigroup on $H$ such that $\left\{T_{t} x: t \in S\right\}$ is bounded for some $x \in C$, let $X$ be a subspace of $B(S)$ such that $1 \in$ $X$ and the mapping $t \rightarrow\left\langle T_{t} x, y^{*}\right\rangle$ is an element of $X$ for each $x \in C$ and $y^{*} \in E^{*}$, and $\mu$ is a mean on $X$. If we write $T_{\mu} x$ instead of $\int T_{t} x d \mu(t)$, then the followings hold.

(i) $T_{\mu}$ is non-expansive mapping from $C$ into $C$.

(ii) $T_{\mu} x=x$ for each $x \in F i x(\phi)$.

(iii) $T_{\mu} x \in \overline{c o}\left\{T_{t} x: t \in S\right\}$ for each $x \in C$.

Let $C$ be a nonempty subset of a Hilbert space $H$ and $T: C \rightarrow H$ a mapping. Then $T$ is said to be demiclosed at $v \in H$ if, for any sequence $\left\{x_{n}\right\}$ in $C$, the following implication holds:

$$
x_{n} \rightarrow u \in C, \quad T x_{n} \rightarrow v \quad \text { imply } \quad T u=v,
$$

where $\rightarrow$ (resp. $\rightarrow$ ) denotes strong (resp. weak) convergence.

Lemma 2.2 [17]Let $C$ be a nonempty closed convex subset of a Hilbert space $H$ and suppose that $T: C \rightarrow H$ is nonexpansive. Then, the mapping $I-T$ is demiclosed at zero.

Lemma 2.3 [18] For a given $x \in H, y \in C$,

$$
y=P_{C} x \Leftrightarrow\langle y-x, z-y\rangle \geq 0, \quad \forall z \in C .
$$


It is well known that $P_{C}$ is a firmly nonexpansive mapping of $H$ onto $C$ and satisfies

$$
\left\|P_{C} x-P_{C} y\right\|^{2} \leq\left\langle P_{C} x-P_{C} y, x-y\right\rangle, \quad \forall x, y \in H
$$

Moreover, $P_{C}$ is characterized by the following properties: $P_{C} x \in C$ and for all $x \in H$, $y \in C$,

$$
\left\langle x-P_{C} x, y-P_{C} x\right\rangle \leq 0 .
$$

It is easy to see that (9) is equivalent to the following inequality

$$
\|x-y\|^{2} \geq\left\|x-P_{C} x\right\|^{2}+\left\|y-P_{C} x\right\|^{2} .
$$

Using Lemma 2.3, one can see that the variational inequality (24) is equivalent to a fixed point problem.

It is easy to see that the following is true:

$$
u \in V I(C, B) \Leftrightarrow u=P_{C}(u-\lambda B u), \quad \lambda>0 .
$$

A set-valued mapping $U: H \rightarrow 2^{H}$ is called monotone if for all $x, y \in H, f \in U x$ and $g \in U y$ imply $\langle x-y, f-g\rangle \geq 0$. A monotone mapping $U: H \rightarrow 2^{H}$ is maximal if the graph of $G(U)$ of $U$ is not properly contained in the graph of any other monotone mapping. It is known that a monotone mapping $U$ is maximal if and only if for $(x, f) \in$ $H \times H,\langle x-y, f-g\rangle \geq 0$ for every $(y, g) \in G(U)$ implies that $f \in U x$. Let $B$ be a monotone mapping of $C$ into $H$ and let $N_{C} x$ be the normal cone to $C$ at $x \in C$, that is, $N_{C} x$ $=\{y \in H:\langle z-x, y\rangle \leq 0, \forall z \in C\}$ and define

$$
U x= \begin{cases}B x+N_{C} x, & x \in C, \\ \emptyset & x \notin C .\end{cases}
$$

Then $U$ is the maximal monotone and $0 \in U x$ if and only if $x \in V I(C, B)$; see [19]. The following lemma is well known.

Lemma 2.4 Let $H$ be a real Hilbert space. Then, for all $x, y \in H$

$$
\|x-y\|^{2} \leq\|x\|^{2}+2\langle y, x+y\rangle, .
$$

Lemma $2.5[5]$ Let $\left\{a_{n}\right\}$ be a sequence of nonnegative real numbers such that

$$
a_{n+1} \leq\left(1-b_{n}\right) a_{n}+b_{n} c_{n}, \quad n \geq 0,
$$

where $\left\{b_{n}\right\}$ and $\left\{c_{n}\right\}$ are sequences of real numbers satisfying the following conditions:

(i) $\left\{b_{n}\right\} \subset(0,1), \sum_{n=0}^{\infty} b_{n}=\infty$,

(ii) either $\limsup _{n \rightarrow \infty} c_{n} \leq 0_{\text {or }} \sum_{n=0}^{\infty}\left|b_{n} c_{n}\right|<\infty$.

Then, $\lim _{n \rightarrow \infty} a_{n}=0$.

As far as we know, the following lemma has been used implicitly in some papers; for the sake of completeness, we include its proof.

Lemma 2.6 Let $H$ be a real Hilbert space and $F$ be a k-Lipschitzian and $\eta$-strongly monotone operator with $k>0, \eta>0$. Let $0<\mu<\frac{2 \eta}{k^{2}}$ and $\tau=\mu\left(\eta-\frac{\mu k^{2}}{2}\right)$. Then for $t \in\left(0, \min \left\{1, \frac{1}{\tau}\right\}\right), I-t_{\mu} F$ is contraction with constant $1-t \tau$. 
Proof. Notice that

$$
\begin{aligned}
\|(I & -t \mu F) x-(I-t \mu F) y \|^{2} \\
& =\langle(I-t \mu F) x-(I-t \mu F) y,(I-t \mu F) x-(I-t \mu F) y\rangle \\
& =\|x-y\|^{2}+t^{2} \mu^{2}\|F x-F y\|^{2}-2 t \mu\langle x-y, F x-F y\rangle \\
& \leq\|x-y\|^{2}+t^{2} \mu^{2} k^{2}\|x-y\|^{2}-2 t \mu \eta\|x-y\|^{2} \\
& \leq\|x-y\|^{2}+t \mu^{2} k^{2}\|x-y\|^{2}-2 t \mu \eta\|x-y\|^{2} \\
& =\left(1-2 t \mu\left(\eta-\frac{\mu k^{2}}{2}\right)\right)\|x-y\|^{2} \\
& =(1-2 t \tau)\|x-y\|^{2} \\
& \leq(1-t \tau)^{2}\|x-y\|^{2} .
\end{aligned}
$$

It follows that

$$
\|(I-t \mu F) x-(I-t \mu F) y\| \leq(1-t \tau)\|x-y\|,
$$

and hence $I-t \mu F$ is contractive due to $1-t \tau \in(0,1)$.

Notation Throughout the rest of this paper, $F$ will denote a $k$-Lipschitzian and $\eta$ strongly monotone operator of $C$ into $H$ with $k>0, \eta>0, f$ is a contraction on $C$ with coefficient $0<\alpha<1$. We will also always use $\gamma$ to mean a number in $\left(0, \frac{\tau}{\alpha}\right)$, where $\tau=\mu\left(\eta-\frac{\mu k^{2}}{2}\right)$ and $0<\mu<\frac{2 \eta}{k^{2}}$. The open ball of radius $r$ centered at 0 is denoted by $B_{r}$ and for a subset $D$ of $H$, by $\overline{c o} D$, we denote the closed convex hull of $D$. For $\varepsilon>0$ and a mapping $T: D \rightarrow H$, we let $F_{\varepsilon}(T ; D)$ be the set of $\varepsilon$-approximate fixed points of $T$, i.e., $F_{\varepsilon}(T ; D)=\left\{x \in D:\left\|x-T_{x}\right\| \leq \varepsilon\right\}$. Weak convergence is denoted by $\rightarrow$ and strong convergence is denoted by $\rightarrow$.

\section{Main results}

Theorem 3.1 Let $S$ be a semigroup, $C$ a nonempty closed convex subset of real Hilbert space $H$ and $B: C \rightarrow H$ be a $\beta$-inverse strongly monotone. Let $\phi=\left\{T_{t}: t \in S\right\}$ be a nonexpansive semigroup of $C$ into itself such that $\mathcal{F}=\operatorname{Fix}(\varphi) \cap V I(C, B) \neq \emptyset_{n}$, X a left invariant subspace of $B(S)$ such that $1 \in X$, and the function $t \rightarrow\left\langle T_{t} x, y\right\rangle$ is an element of $X$ for each $x \in C$ and $y \in H,\left\{\mu_{n}\right\}$ a left regular sequence of means on $X$ such that $\sum_{n=1}^{\infty}\left\|\mu_{n+1}-\mu_{n}\right\|<\infty$. Let $\left\{\alpha_{n}\right\}$ and $\left\{\beta_{n}\right\}$ be sequences in $(0,1)$ and $\left\{\delta_{n}\right\}$ be a sequence in $[a, b]$, where $0<a<b<2 \beta$. Suppose the following conditions are satisfied.

$\left(B_{1}\right) \lim _{n \rightarrow \infty} \alpha_{n}=0, \lim _{n \rightarrow \infty} \beta_{n}=0$,

$\left(B_{2}\right) \sum_{n=1}^{\infty} \alpha_{n}=\infty$,

$\left(B_{3}\right) \sum_{n=1}^{\infty}\left|\alpha_{n+1}-\alpha_{n}\right|<\infty, \sum_{n=1}^{\infty}\left|\beta_{n+1}-\beta_{n}\right|<\infty, \sum_{n=1}^{\infty}\left|\delta_{n+1}-\delta_{n}\right|<\infty$.

If $\left\{x_{n}\right\}$ and $\left\{y_{n}\right\}$ be generated by $x_{0} \in C$ and

$$
\left\{\begin{array}{l}
y_{n}=\beta_{n} x_{n}+\left(1-\beta_{n}\right) P_{C}\left(x_{n}-\delta_{n} B x_{n}\right), \\
x_{n+1}=\alpha_{n} \gamma f\left(x_{n}\right)+\left(I-\alpha_{n} \mu F\right) T_{\mu_{n}} y_{n}, \quad n \geq 0 .
\end{array}\right.
$$

Then, $\left\{x_{n}\right\}$ and $\left\{y_{n}\right\}$ converge strongly, as $n \rightarrow \infty$, to $x^{*} \in \mathcal{F}$, which is a unique solution of the variational inequalities: 


$$
\begin{cases}\left\langle(\mu F-\gamma f) x^{*}, x-x^{*}\right\rangle \geq 0, & \forall x \in \mathcal{F} \\ \left\langle B x^{*}, y-x^{*}\right\rangle \geq 0 & \forall y \in C\end{cases}
$$

Proof. Since $\left\{\alpha_{n}\right\}$ satisfies in condition $\left(B_{1}\right)$, we may assume, with no loss of generality, that $\alpha_{n}<\min \left\{1, \frac{1}{\tau}\right\}$. Since $B$ is $\beta$-inverse strongly monotone and $\delta_{n}<2 \beta$, for any $x, y \in C$, we have

$$
\begin{aligned}
\| & \left(I-\delta_{n} B\right) x-\left(I-\delta_{n} B\right) y \|^{2} \\
& =\left\|(x-y)-\delta_{n}(B x-B y)\right\|^{2} \\
& =\|x-y\|^{2}-2 \delta_{n}\langle x-y, B x-B y\rangle+\delta_{n}^{2}\|B x-B y\|^{2} \\
& \leq\|x-y\|^{2}-2 \delta_{n} \beta\|B x-B y\|^{2}+\delta_{n}^{2}\|B x-B y\|^{2} \\
& =\|x-y\|^{2}+\delta_{n}\left(\delta_{n}-2 \beta\right)\|B x-B y\|^{2} \\
& \leq\|x-y\|^{2} .
\end{aligned}
$$

It follows that

$$
\left\|\left(I-\delta_{n} B\right) x-\left(I-\delta_{n} B\right) y\right\| \leq\|x-y\| .
$$

Let $p \in \mathcal{F}$, in the context of the variational inequality problem, the characterization of projection (11) implies that $p=P_{C}\left(p-\delta_{n} B_{p}\right)$. Using (13), we get

$$
\begin{aligned}
\left\|y_{n}-p\right\| & =\left\|\beta_{n} x_{n}+\left(1-\beta_{n}\right) P_{C}\left(x_{n}-\delta_{n} B x_{n}\right)-p\right\| \\
& =\left\|\beta_{n}\left[x_{n}-p\right]+\left(1-\beta_{n}\right)\left[P_{C}\left(x_{n}-\delta_{n} B x_{n}\right)-P_{C}\left(p-\delta_{n} B p\right)\right]\right\| \\
& \leq \beta_{n}\left\|x_{n}-p\right\|+\left(1-\beta_{n}\right)\left\|P_{C}\left(x_{n}-\delta_{n} B x_{n}\right)-P_{C}\left(p-\delta_{n} B p\right)\right\| \\
& \leq \beta_{n}\left\|x_{n}-p\right\|+\left(1-\beta_{n}\right)\left\|x_{n}-p\right\|=\left\|x_{n}-p\right\| .
\end{aligned}
$$

We claim that $\left\{x_{n}\right\}$ is bounded. Let $p \in \mathcal{F}$, using Lemma 2.6 and (14), we have

$$
\begin{aligned}
\left\|x_{n+1}-p\right\|= & \left\|\alpha_{n} \gamma f\left(x_{n}\right)+\left(I-\alpha_{n} \mu F\right) T_{\mu_{n}} y_{n}-p\right\| \\
= & \left\|\alpha_{n} \gamma f\left(x_{n}\right)+\left(I-\alpha_{n} \mu F\right) T_{\mu_{n}} y_{n}-\left(I-\alpha_{n} \mu F\right) p-\alpha_{n} \mu F p\right\| \\
\leq & \alpha_{n}\left\|\gamma f\left(x_{n}\right)-\mu F p\right\|+\left\|\left(I-\alpha_{n} \mu F\right) T_{\mu_{n}} y_{n}-\left(I-\alpha_{n} \mu F\right) p\right\| \\
\leq & \alpha_{n}\left\|\gamma f\left(x_{n}\right)-\gamma f(p)\right\| \\
& +\alpha_{n}\|\gamma f(p)-\mu F p\|+\left(1-\alpha_{n} \tau\right)\left\|T_{\mu_{n}} y_{n}-p\right\| \\
\leq & \alpha_{n} \gamma \alpha\left\|x_{n}-p\right\|+\alpha_{n}\|\gamma f(p)-\mu F p\|+\left(1-\alpha_{n} \tau\right)\left\|y_{n}-p\right\| \\
\leq & \alpha_{n} \gamma \alpha\left\|x_{n}-p\right\|+\alpha_{n}\|\gamma f(p)-\mu F p\|+\left(1-\alpha_{n} \tau\right)\left\|x_{n}-p\right\| \\
= & \left(1-\alpha_{n}(\tau-\gamma \alpha)\right)\left\|x_{n}-p\right\|+\alpha_{n}\|\gamma f(p)-\mu F p\| \\
\leq & \max \left\{\left\|x_{n}-p\right\|,(\tau-\gamma \alpha)^{-1}\|\gamma f(p)-\mu F p\|\right\} .
\end{aligned}
$$

By induction we have,

$$
\left\|x_{n}-p\right\| \leq \max \left\{(\tau-\gamma \alpha)^{-1}\|\gamma f(p)-\mu F p\|,\left\|x_{0}-p\right\|\right\}=M_{0} .
$$

Hence, $\left\{x_{n}\right\}$ is bounded and also $\left\{y_{n}\right\}$ and $\left\{f\left(x_{n}\right)\right\}$ are bounded. Set $D=\{y \in H: \| y$ $\left.p \| \leq=M_{0}\right\}$. We remark that $D$ is $\phi$-invariant bounded closed convex set and $\left\{x_{n}\right\},\left\{y_{n}\right\}$ $\subset D$. Now we claim that

$$
\limsup _{n \rightarrow \infty} \sup _{\gamma \in D}\left\|T_{\mu_{n}} y-T_{t} T_{\mu_{n}} y\right\|=0, \quad \forall t \in S .
$$

Let $\varepsilon>0$. By [[20], Theorem 1.2], there exists $\delta>0$ such that

$$
\overline{c o} F_{\delta}\left(T_{t} ; D\right)+B_{\delta} \subset F_{\varepsilon}\left(T_{t} ; D\right), \quad \forall t \in S .
$$


Also by [[20], Corollary 1.1], there exists a natural number $N$ such that

$$
\left\|\frac{1}{N+1} \sum_{i=0}^{N} T_{t^{i} s} y-T_{t}\left(\frac{1}{N+1} \sum_{i=0}^{N} T_{t^{i}} y\right)\right\| \leq \delta,
$$

for all $t, s \in S$ and $y \in D$. Let $t \in S$. Since $\left\{\mu_{n}\right\}$ is strongly left regular, there exists $N_{0}$ $\in \mathbb{N}$ such that $\left\|\mu_{n}-l_{t^{i}}^{*} \mu_{n}\right\| \leq \frac{\delta}{\left(M_{0}+\|p\|\right)}$ for $n \geq N_{0}$ and $i=0,1,2, \ldots, N$. Then we have

$$
\begin{aligned}
\sup _{y \in D} \| & T_{\mu_{n}} y-\int \frac{1}{N+1} \sum_{i=0}^{N} T_{t^{i} s} \gamma \mathrm{d} \mu_{n} s \| \\
& =\sup _{\gamma \in D} \sup _{\|z\|=1}\left|\left\langle T_{\mu_{n}} y, z\right\rangle-\left\langle\int \frac{1}{N+1} \sum_{i=0}^{N} T_{t^{i} s} \gamma \mathrm{d} \mu_{n} s, z\right\rangle\right| \\
& =\sup _{\gamma \in D} \sup _{\|z\|=1}\left|\frac{1}{N+1} \sum_{i=0}^{N}\left(\mu_{n}\right)_{s}\left\langle T_{s} y, z\right\rangle-\frac{1}{N+1} \sum_{i=0}^{N}\left(\mu_{n}\right)_{s}\left\langle T_{t^{i} s} y, z\right\rangle\right| \\
& \leq \frac{1}{N+1} \sum_{i=0}^{N} \sup _{\gamma \in D} \sup _{\|z\|=1}\left|\left(\mu_{n}\right)_{s}\left\langle T_{s} y, z\right\rangle-\left(l_{t^{i}}^{*} \mu_{n}\right)_{s}\left\langle T_{s} y, z\right\rangle\right| \\
& \leq \max _{i=1,2, \ldots N}\left\|\mu_{n}-l_{t^{i}}^{*} \mu_{n}\right\|\left(M_{0}+\|p\|\right) \leq \delta, \forall n \geq N_{0} .
\end{aligned}
$$

By Lemma 2.1, we have

$$
\int \frac{1}{N+1} \sum_{i=0}^{N} T_{t^{i} s} \gamma \mathrm{d} \mu_{n} s \in \overline{c o}\left\{\frac{1}{N+1} \sum_{i=0}^{N} T_{t^{i}}\left(T_{s} y\right): s \in s\right\} .
$$

It follows from (16), (17), (18), and (19) that

$$
\begin{aligned}
T_{\mu_{n}}(y) & \in \overline{c o}\left\{\frac{1}{N+1} \sum_{i=0}^{N} T_{t^{i} s}(y): s \in S\right\}+B_{\delta} \\
& \subset \overline{c o} F_{\delta}\left(T_{t} ; D\right)+B_{\delta} \subset F_{\varepsilon}\left(T_{t} ; D\right),
\end{aligned}
$$

for all $y \in D$ and $n \geq N_{0}$. Therefore,

$$
\limsup _{n \rightarrow \infty} \sup _{\gamma \in D}\left\|T_{t}\left(T_{\mu_{n}} y\right)-T_{\mu_{n}} y\right\| \leq \varepsilon
$$

Since $\varepsilon>0$ is arbitrary, we get (15). In this stage, we will show

$$
\lim _{n \rightarrow \infty}\left\|x_{n}-T_{t} x_{n}\right\|=0, \quad \forall t \in S .
$$

Let $t \in S$ and $\varepsilon>0$. Then, there exists $\delta>0$, which satisiies (16). From $\lim _{n \rightarrow \infty} \alpha_{n}=$ 0 and (15) there exists $N_{1} \in \mathbb{N}$ such that $\alpha_{n} \leq \frac{\delta}{(\tau+\mu k) M_{0}}$ and $T_{\mu_{n}} y_{n} \in F_{\delta}\left(T_{t} ; D\right)$, for all $n$ $\geq N_{1}$. By Lemma 2.6 and (14), we have

$$
\begin{aligned}
\alpha_{n} \| & \gamma f\left(x_{n}\right)-\mu F T_{\mu_{n}} y_{n} \| \\
& \leq \alpha_{n}\left(\gamma\left\|f\left(x_{n}\right)-f(p)\right\|+\|\gamma f(p)-\mu F p\|+\left\|\mu F p-\mu F T_{\mu_{n}} y_{n}\right\|\right) \\
& \leq \alpha_{n}\left(\gamma \alpha\left\|x_{n}-p\right\|+\|\gamma f(p)-\mu F p\|+\mu k\left\|y_{n}-p\right\|\right) \\
& \leq \alpha_{n}\left(\gamma \alpha M_{0}+(\tau-\gamma \alpha) M_{0}+\mu k M_{0}\right) \\
& \leq \alpha_{n}(\tau+\mu k) M_{0} \leq \delta,
\end{aligned}
$$


for all $n \geq N_{1}$. Therefore, we have

$$
\begin{aligned}
x_{n+1} & =T_{\mu_{n}} y_{n}+\alpha_{n}\left[\gamma f\left(x_{n}\right)+\mu F\left(T_{\mu_{n}} y_{n}\right)\right] \\
& \in F_{\delta}\left(T_{t} ; D\right)+B_{\delta} \subset F_{\varepsilon}\left(T_{t} ; D\right),
\end{aligned}
$$

for all $n \geq N_{1}$. This shows that

$$
\left\|x_{n}-T_{t} x_{n}\right\| \leq \varepsilon, \forall n \geq N_{1} .
$$

Since $\varepsilon>0$ is arbitrary, we get (20).

Let

$Q=P_{\mathcal{F}}$. Then $Q(I-\mu F+\gamma f)$ is a contraction of $H$ into itself. In fact, we see that

$$
\begin{aligned}
& \|Q(I-\mu F+\gamma f) x-Q(I-\mu F+\gamma f) y\| \\
& \quad \leq\|(I-\mu F+\gamma f) x-(I-\mu F+\gamma f) y\| \\
& \quad \leq\|(I-\mu F) x-(I-\mu F) y\|+\gamma\|f(x)-f(\gamma)\| \\
& \quad=\lim _{n \rightarrow \infty}\left\|\left(I-\left(1-\frac{1}{n}\right) \mu F\right) x-\left(I-\left(1-\frac{1}{n}\right) \mu F\right) \gamma\right\|+\gamma\|f(x)-f(\gamma)\| \\
& \quad \leq \lim _{n \rightarrow \infty}\left(1-\left(1-\frac{1}{n}\right) \tau\right)\|x-y\|+\gamma \alpha\|x-\gamma\| \\
& \quad=(1-\tau)\|x-\gamma\|+\gamma \alpha\|x-\gamma\|,
\end{aligned}
$$

and hence $Q(I-\mu F+\gamma f)$ is a contraction due to $(1-(\tau-\gamma \alpha)) \in(0,1)$.

Therefore, by Banachs contraction principal, $P_{\mathcal{F}}(I-\mu F+\gamma f)$ has a unique fixed point $x^{*}$. Then using (9), $x^{*}$ is the unique solution of the variational inequality:

$$
\left\langle(\gamma f-\mu F) x^{*}, x-x^{*}\right\rangle \leq 0, \quad \forall x \in \mathcal{F} .
$$

We show that

$$
\limsup _{n \rightarrow \infty}\left\langle\gamma f\left(x^{*}\right)-\mu F x^{*}, x_{n}-x^{*}\right\rangle \leq 0 .
$$

Indeed, we can choose a subsequence $\left\{x_{n_{i}}\right\}$ of $\left\{x_{n}\right\}$ such that

$$
\limsup _{n \rightarrow \infty}\left\langle\gamma f\left(x^{*}\right)-\mu F x^{*}, x_{n}-x^{*}\right\rangle=\lim _{i \rightarrow \infty}\left\langle\gamma f\left(x^{*}\right)-\mu F x^{*}, x_{n_{i}}-x^{*}\right\rangle .
$$

Because $\left\{x_{n_{i}}\right\}$ is bounded, we may assume that $x_{n_{i}} \rightarrow z$. In terms of Lemma 2.2 and (20), we conclude that $z \in$ Fix $(\phi)$.

Now, let us show that $z \in V I(C, B)$. Let $w_{n}=P_{C}\left(x_{n}-\delta_{n} B x_{n}\right)$, it follows from the definition of $\left\{y_{n}\right\}$ that

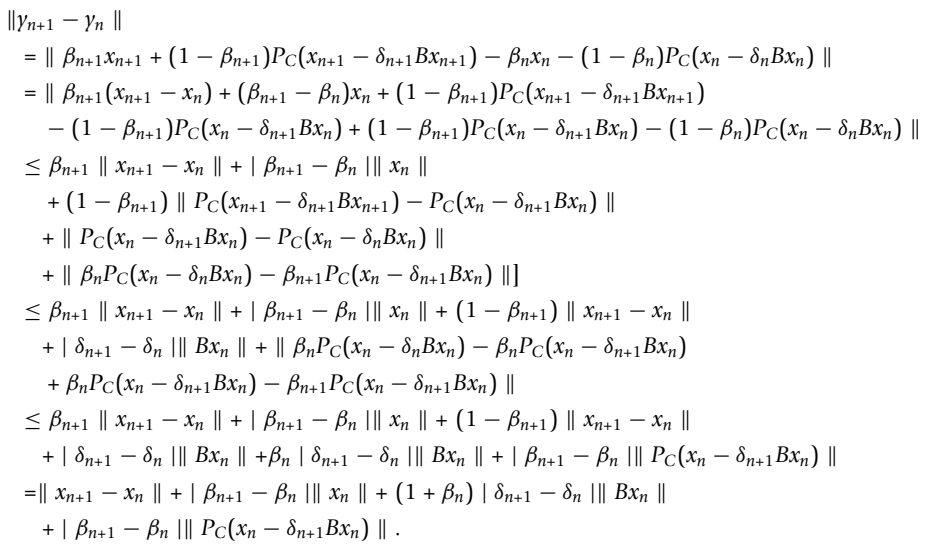


Using the last inequality, we get

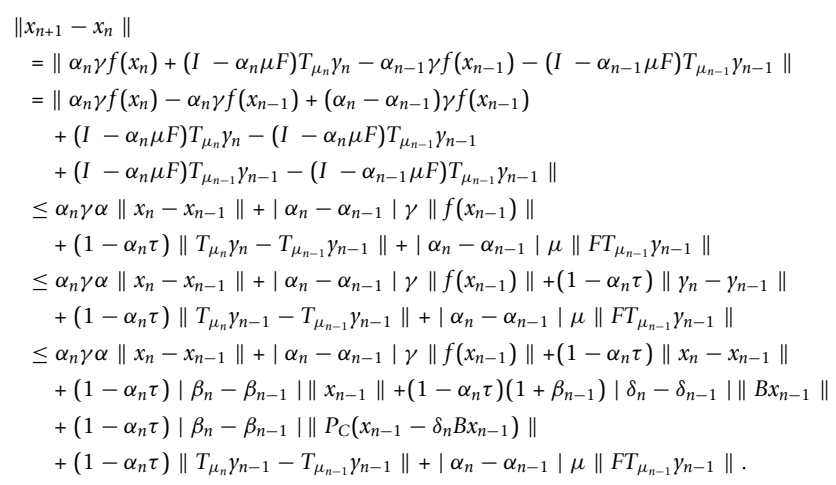

Thus, for some large enough constant $M>0$, we have

$$
\begin{aligned}
\left\|x_{n+1}-x_{n}\right\| \leq & \left(1-\alpha_{n}(\tau-\gamma \alpha)\right)\left\|x_{n}-x_{n-1}\right\| \\
& +\left[\left|\alpha_{n}-\alpha_{n-1}\right|+\left|\beta_{n}-\beta_{n-1}\right|+\left|\delta_{n}-\delta_{n-1}\right|+\left\|\mu_{n}-\mu_{n-1}\right\|\right] M .
\end{aligned}
$$

Therefore, using condition $B_{3}$ and Lemma 2.5, we get

$$
\lim _{n \rightarrow \infty}\left\|x_{n+1}-x_{n}\right\|=0 .
$$

Let $p \in \mathcal{F}$, from (11) and deiinition of $\left\{y_{n}\right\}$, we have

$$
\begin{aligned}
&\left\|y_{n}-p\right\|^{2} \\
&=\left\|\beta_{n} x_{n}+\left(1-\beta_{n}\right) P_{C}\left(x_{n}-\delta_{n} B x_{n}\right)-p\right\|^{2} \\
&=\left\|\beta_{n}\left(x_{n}-p\right)+\left(1-\beta_{n}\right)\left(P_{C}\left(x_{n}-\delta_{n} B x_{n}\right)-P_{C}\left(p-\delta_{n} B p\right)\right)\right\|^{2} \\
&\left.\leq \beta_{n}\left\|x_{n}-p\right\|^{2}+\left(1-\beta_{n}\right) \|\left(x_{n}-p\right)-\delta_{n}\left(B x_{n}-B p\right)\right) \|^{2} \\
&=\beta_{n}\left\|x_{n}-p\right\|^{2}+\left(1-\beta_{n}\right)\left\|x_{n}-p\right\|^{2}+\delta_{n}^{2}\left(1-\beta_{n}\right)\left\|B x_{n}-B p\right\|^{2} \\
& \quad-2 \delta_{n}\left(1-\beta_{n}\right)\left\langle x_{n}-p, B x_{n}-B p\right\rangle \\
& \leq\left\|x_{n}-p\right\|^{2}+\delta_{n}^{2}\left(1-\beta_{n}\right)\left\|B x_{n}-B p\right\|^{2}-2 \delta_{n}\left(1-\beta_{n}\right) \beta\left\|B x_{n}-B p\right\|^{2} \\
&=\left\|x_{n}-p\right\|^{2}+\delta_{n}\left(1-\beta_{n}\right)\left(\delta_{n}-2 \beta\right)\left\|B x_{n}-B p\right\|^{2} .
\end{aligned}
$$

Using (25), we have

$$
\begin{aligned}
&\left\|x_{n+1}-p\right\|^{2} \\
&=\left\|\alpha_{n} \gamma f\left(x_{n}\right)+\left(I-\alpha_{n} \mu F\right) T_{\mu_{n}} y_{n}-p\right\|^{2} \\
&=\left\|\alpha_{n}\left(\gamma f\left(x_{n}\right)-\mu F T_{\mu_{n}} y_{n}\right)+\left(T_{\mu_{n}} y_{n}-p\right)\right\|^{2} \\
&= \alpha_{n}^{2}\left\|\gamma f\left(x_{n}\right)-\mu F T_{\mu_{n}} y_{n}\right\|^{2}+\left\|T_{\mu_{n}} y_{n}-p\right\| \\
&+2 \alpha_{n}\left\langle\gamma f\left(x_{n}\right)-\mu F T_{\mu_{n}} y_{n}, T_{\mu_{n}} y_{n}-p\right\rangle \\
& \leq \alpha_{n}^{2}\left\|\gamma f\left(x_{n}\right)-\mu F T_{\mu_{n}} y_{n}\right\|^{2}+\left\|y_{n}-p\right\|^{2} \\
&+2 \alpha_{n}\left\langle\gamma f\left(x_{n}\right)-\mu F T_{\mu_{n}} y_{n}, T_{\mu_{n}} y_{n}-p\right\rangle^{2} \\
& \leq \alpha_{n}^{2}\left\|\gamma f\left(x_{n}\right)-\mu F T_{\mu_{n}} y_{n}\right\|^{2}+\left\|x_{n}-p\right\|^{2} \\
&+\delta_{n}\left(1-\beta_{n}\right)\left(\delta_{n}-2 \beta\right)\left\|B x_{n}-B p\right\|^{2} \\
&+2 \alpha_{n}\left\langle\gamma f\left(x_{n}\right)-\mu F T_{\mu_{n}} y_{n}, T_{\mu_{n}} y_{n}-p\right\rangle \\
&= \alpha_{n}^{2}\left\|\gamma f\left(x_{n}\right)-\mu F T_{\mu_{n}} y_{n}\right\|^{2}+\left\|x_{n}-p\right\|^{2} \\
&+\delta_{n}\left(\delta_{n}-2 \beta_{n}\right)\left\|B x_{n}-B p\right\|^{2}-\delta_{n} \beta_{n}\left(\delta_{n}-2 \beta_{n}\right)\left\|B x_{n}-B p\right\|^{2} \\
&+2 \alpha_{n}\left\langle\gamma f\left(x_{n}\right)-\mu F T_{\mu_{n}} y_{n}, T_{\mu_{n}} y_{n}-p\right\rangle .
\end{aligned}
$$


Therefore,

$$
\begin{aligned}
& -\delta_{n}\left(\delta_{n}-2 \beta_{n}\right)\left\|B x_{n}-B p\right\|^{2} \\
& \quad \leq \alpha_{n}^{2}\left\|\gamma f\left(x_{n}\right)-\mu F T_{\mu_{n}} y_{n}\right\|^{2}+\left[\left\|x_{n}-p\right\|+\left\|x_{n+1}-p\right\|\right]\left\|x_{n+1}-x_{n}\right\| \\
& \quad-\delta_{n} \beta_{n}\left(\delta_{n}-2 \beta_{n}\right)\left\|B x_{n}-B p\right\|^{2}+2 \alpha_{n}\left\langle\gamma f\left(x_{n}\right)-\mu F T_{\mu_{n}} y_{n}, T_{\mu_{n}} y_{n}-p\right\rangle .
\end{aligned}
$$

Hence, using condition $B_{1}$ and (24), we get

$$
\lim _{n \rightarrow \infty}\left\|B x_{n}-B p\right\|=0 .
$$

From (8), we have

$$
\begin{aligned}
&\left\|w_{n}-p\right\|^{2} \\
&=\left\|P_{C}\left(x_{n}-\delta_{n} B x_{n}\right)-P_{C}\left(p-\delta_{n} B p\right)\right\|^{2} \\
& \leq\left\langle\left(x_{n}-\delta_{n} B x_{n}\right)-\left(p-\delta_{n} B p\right), w_{n}-p\right\rangle \\
&= \frac{1}{2}\left[\left\|\left(x_{n}-\delta_{n} B x_{n}\right)-\left(p-\delta_{n} B p\right)\right\|^{2}+\left\|w_{n}-p\right\|^{2}\right. \\
&\left.-\left\|\left(x_{n}-\delta_{n} B x_{n}\right)-\left(p-\delta_{n} B p\right)-\left(w_{n}-p\right)\right\|^{2}\right] \\
& \leq \frac{1}{2}\left[\left\|x_{n}-p\right\|^{2}+\left\|w_{n}-p\right\|^{2}\right. \\
&\left.-\left\|\left(x_{n}-\delta_{n} B x_{n}\right)-\left(p-\delta_{n} B p\right)-\left(w_{n}-p\right)\right\|^{2}\right] \\
&= \frac{1}{2}\left[\left\|x_{n}-p\right\|^{2}+\left\|w_{n}-p\right\|^{2}-\left\|x_{n}-w_{n}\right\|^{2}\right. \\
&\left.+2 \delta_{n}\left\langle x_{n}-w_{n}, B x_{n}-B p\right\rangle-\delta_{n}^{2}\left\|B x_{n}-B p\right\|^{2}\right] .
\end{aligned}
$$

So we obtain

$$
\begin{aligned}
\left\|w_{n}-p\right\|^{2} \leq & \left\|x_{n}-p\right\|^{2}-\left\|x_{n}-w_{n}\right\|^{2} \\
& +2 \delta_{n}\left\langle x_{n}-w_{n}, B x_{n}-B p\right\rangle-\delta_{n}^{2}\left\|B x_{n}-B p\right\|^{2} .
\end{aligned}
$$

It follows from (26) and (28) that

$$
\begin{aligned}
\| x_{n+} & -p \|^{2} \\
\leq & \alpha_{n}^{2}\left\|\gamma f\left(x_{n}\right)-\mu F T_{\mu_{n}} y_{n}\right\|^{2}+\left\|y_{n}-p\right\|^{2} \\
& +2 \alpha_{n}\left\langle\gamma f\left(x_{n}\right)-\mu F T_{\mu_{n}} y_{n}, T_{\mu_{n}} y_{n}-p\right\rangle \\
\leq & \alpha_{n}^{2}\left\|\gamma f\left(x_{n}\right)-\mu F T_{\mu_{n}} y_{n}\right\|^{2}+\left\|\beta_{n} x_{n}+\left(1-\beta_{n}\right) P_{C}\left(x_{n}-\delta_{n} B x_{n}\right)-p\right\|^{2} \\
& +2 \alpha_{n}\left\langle\gamma f\left(x_{n}\right)-\mu F T_{\mu_{n}} y_{n}, T_{\mu_{n}} y_{n}-p\right\rangle \\
\leq & \alpha_{n}^{2}\left\|\gamma f\left(x_{n}\right)-\mu F T_{\mu_{n}} y_{n}\right\|^{2}+\beta_{n}\left\|x_{n}-p\right\|^{2}+\left(1-\beta_{n}\right)\left\|w_{n}-p\right\|^{2} \\
& +2 \alpha_{n}\left\langle\gamma f\left(x_{n}\right)-\mu F T_{\mu_{n}} y_{n}, T_{\mu_{n}} y_{n}-p\right\rangle \\
\leq & \alpha_{n}^{2}\left\|\gamma f\left(x_{n}\right)-\mu F T_{\mu_{n}} y_{n}\right\|^{2}+\beta_{n}\left\|x_{n}-p\right\|^{2}+\left(1-\beta_{n}\right)\left\|x_{n}-p\right\|^{2} \\
& -\left(1-\beta_{n}\right)\left\|x_{n}-w_{n}\right\|^{2}+2 \delta_{n}\left(1-\beta_{n}\right)\left\langle x_{n}-w_{n}, B x_{n}-B p\right\rangle-\delta_{n}^{2}\left(1-\beta_{n}\right)\left\|B x_{n}-B p\right\|^{2} \\
& +2 \alpha_{n}\left\langle\gamma f\left(x_{n}\right)-\mu F T_{\mu_{n}} y_{n}, T_{\mu_{n}} y_{n}-p\right\rangle .
\end{aligned}
$$

Which implies that

$$
\begin{aligned}
\left\|x_{n}-w_{n}\right\|^{2} \leq & \alpha_{n}^{2}\left\|\gamma f\left(x_{n}\right)-\mu F T_{\mu_{n}} y_{n}\right\|^{2}+\left[\left\|x_{n}-p\right\|+\left\|x_{n+1}-p\right\|\right]\left\|x_{n+1}-x_{n}\right\| \\
& +\beta_{n}\left\|x_{n}-w_{n}\right\|^{2}+2 \delta_{n}\left(1-\beta_{n}\right)\left\|x_{n}-w_{n}\right\|\left\|B x_{n}-B p\right\| \\
& -\delta_{n}^{2}\left(1-\beta_{n}\right)\left\|B x_{n}-B p\right\|^{2}+2 \alpha_{n}\left\langle\gamma f\left(x_{n}\right)-\mu F T_{\mu_{n}} y_{n}, T_{\mu_{n}} y_{n}-p\right\rangle .
\end{aligned}
$$


Therefore, using condition $B_{1}$, (24) and (27), we get

$$
\lim _{n \rightarrow \infty}\left\|x_{n}-w_{n}\right\|=0 .
$$

Let $U: H \rightarrow 2^{H}$ be a set-valued mapping is defined by

$$
U x= \begin{cases}A x+N_{C} x, & x \in C, \\ \emptyset, & x \notin C .\end{cases}
$$

where $N_{C} x$ is the normal cone to $C$ at $x \in C$. Since $B$ is relaxed, $\beta$-inverse strongly monotone. Thus, $U$ is maximal monotone see [19]. Let $(x, y) \in G(U)$, hence $y-B x \in$ $N_{C} x$. Since $w_{n}=P_{C}\left(x_{n}-\zeta_{n} B x_{n}\right)$ therefore, $\left\langle x-w_{n}, y-B x\right\rangle \geq 0$. On the other hand from $w_{n}=P_{C}\left(x_{n}-\zeta_{n} B x_{n}\right)$, we have

$$
\left\langle x-w_{n}, w_{n}-\left(x_{n}-\delta_{n} B x_{n}\right)\right\rangle \geq 0,
$$

that is

$$
\left\langle x-w_{n}, \frac{w_{n}-x_{n}}{\delta_{n}}+B x_{n}\right\rangle \geq 0 .
$$

Therefore, we have

$$
\begin{aligned}
\langle x & \left.-w_{n_{i}}, y\right\rangle \\
& \geq\left\langle x-w_{n_{i}}, B x\right\rangle \\
& \left.\geq\left\langle x-w_{n_{i}}, B x\right\rangle-\left\langle x-w_{n_{i}}, \frac{w_{n_{i}}-x_{n_{i}}}{\delta_{n_{i}}}+B x_{n_{i}}\right)\right\rangle \\
& =\left\langle x-w_{n_{i}}, B x-\frac{w_{n_{i}}-x_{n_{i}}}{\delta_{n_{i}}}-B x_{n_{i}}\right\rangle \\
& =\left\langle x-w_{n_{i}}, B x-B w_{n_{i}}\right\rangle+\left\langle x-w_{n_{i}}, B w_{n_{i}}-B x_{n_{i}}\right\rangle-\left\langle x-w_{n_{i}}, \frac{w_{n_{i}}-x_{n_{i}}}{\delta_{n_{i}}}\right\rangle \\
& \geq\left\langle x-w_{n_{i}}, B w_{n_{i}}-B x_{n_{i}}\right\rangle-\left\langle x-w_{n_{i}}, \frac{w_{n_{i}}-x_{n_{i}}}{\delta_{n_{i}}}\right\rangle \\
& \geq\left\langle x-w_{n_{i}}, B w_{n_{i}}-B x_{n_{i}}\right\rangle-\left\|x-w_{n_{i}}\right\|\left\|\frac{w_{n_{i}}-x_{n_{i}}}{\delta_{n_{i}}}\right\| .
\end{aligned}
$$

Noting that $\lim _{i \rightarrow \infty}\left\|w_{n_{i}}-x_{n_{i}}\right\|=0, x_{n_{i}} \rightarrow z, x_{n_{i}} \rightarrow z$ and $B$ is $\frac{1}{\beta}$-lipschitzian, we obtain

$$
\langle x-z, y\rangle \geq 0
$$

Since $U$ is maximal monotone, we have $z \in U^{-1} 0$, and hence $z \in V I(C, B)$. Therefore, $z \in \mathcal{F}$.

Since $x_{n_{i}} \rightarrow z$ from (21) and (23), we have

$$
\limsup _{n \rightarrow \infty}\left\langle\gamma f\left(x^{*}\right)-\mu F x^{*}, x_{n}-x^{*}\right\rangle \leq 0 .
$$


Finally, we prove that $x_{n} \rightarrow x^{*}$ as $n \rightarrow \infty$. By Lemmas 2.4, 2.6, and (14), we have

$$
\begin{aligned}
&\left\|x_{n+1}-x^{*}\right\|^{2} \\
&=\left\|\alpha_{n} \gamma f\left(x_{n}\right)+\left(I-\alpha_{n} \mu F\right) T_{\mu_{n}} y_{n}-x^{*}\right\|^{2} \\
&= \| \alpha_{n} \gamma f\left(x_{n}\right)-\alpha_{n} \mu F x^{*}+\left(I-\alpha_{n} \mu F\right) T_{\mu_{n}} y_{n} \\
&-\left(I-\alpha_{n} \mu F\right) x^{*} \|^{2} \\
& \leq\left\|\left(I-\alpha_{n} \mu F\right) T_{\mu_{n}} y_{n}-\left(I-\alpha_{n} \mu F\right) x^{*}\right\|^{2} \\
&+2 \alpha_{n}\left\langle\gamma f\left(x_{n}\right)-\mu F x^{*}, x_{n+1}-x^{*}\right\rangle \\
& \leq\left(1-\alpha_{n} \tau\right)^{2}\left\|y_{n}-x^{*}\right\|^{2}+2 \alpha_{n}\left\langle\gamma f\left(x_{n}\right)-\mu F x^{*}, x_{n+1}-x^{*}\right\rangle \\
& \leq\left(1-\alpha_{n} \tau\right)^{2}\left\|y_{n}-x^{*}\right\|^{2}+2 \alpha_{n}\left\langle\gamma f\left(x_{n}\right)-\gamma f\left(x^{*}\right), x_{n+1}-x^{*}\right\rangle \\
&+2 \alpha_{n}\left\langle\gamma f\left(x^{*}\right)-\mu F x^{*}, x_{n+1}-x^{*}\right\rangle . \\
& \leq\left(1-\alpha_{n} \tau\right)^{2}\left\|y_{n}-x^{*}\right\|^{2}+\alpha_{n} \gamma \alpha\left[\left\|x_{n}-x^{*}\right\|^{2}+\left\|x_{n+1}-x^{*}\right\|^{2}\right] \\
&+2 \alpha_{n}\left\langle\gamma f\left(x^{*}\right)-\mu F x^{*}, x_{n+1}-x^{*}\right\rangle . \\
& \leq\left(1-\alpha_{n} \tau\right)^{2}\left\|x_{n}-x^{*}\right\|^{2}+\alpha_{n} \gamma \alpha\left[\left\|x_{n}-x^{*}\right\|^{2}+\left\|x_{n+1}-x^{*}\right\|^{2}\right] \\
&+2 \alpha_{n}\left\langle\gamma f\left(x^{*}\right)-\mu F x^{*}, x_{n+1}-x^{*}\right\rangle .
\end{aligned}
$$

So from (30), we reach the following

$$
\begin{aligned}
\left\|x_{n+1}-x^{*}\right\|^{2} \leq & \frac{1+\alpha^{2} \tau^{2}-2 \alpha_{n} \tau+\alpha_{n} \gamma \alpha}{1-\alpha_{n} \gamma \alpha}\left\|x_{n}-x^{*}\right\|^{2} \\
& +\frac{2 \alpha_{n}}{1-\alpha_{n} \gamma \alpha}\left\langle\gamma f\left(x^{*}\right)-\mu F x^{*}, x_{n+1}-x^{*}\right\rangle \\
\leq & \left(1-\alpha_{n} \frac{2(\tau-\gamma \alpha)-\alpha_{n} \tau^{2}}{1-\alpha_{n} \gamma \alpha}\right)\left\|x_{n}-x^{*}\right\|^{2} \\
& +\alpha_{n} \frac{2(\tau-\gamma \alpha)-\alpha_{n} \tau^{2}}{1-\alpha_{n} \gamma \alpha} \frac{2}{2(\tau-\gamma \alpha)-\alpha_{n} \tau^{2}}\left\langle\gamma f\left(x^{*}\right)-\mu F x^{*}, x_{n+1}-x^{*}\right\rangle
\end{aligned}
$$

It follows that

$$
\left\|x_{n+1}-x^{*}\right\|^{2} \leq\left(1-b_{n}\right)\left\|x_{n}-x^{*}\right\|^{2}+b_{n} c_{n},
$$

where

$$
b_{n}=\alpha_{n} \frac{2(\tau-\gamma \alpha)-\alpha_{n} \tau^{2}}{1-\alpha_{n} \gamma \alpha}, c_{n}=\frac{2}{2(\tau-\gamma \alpha)-\alpha_{n} \tau^{2}}\left\langle\gamma f\left(x^{*}\right)-\mu F x^{*}, x_{n+1}-x^{*}\right\rangle
$$

Since $\alpha_{n} \rightarrow \mathrm{o}$ and $\sum_{n=0}^{\infty} \alpha_{n}=\infty$, we have $\sum_{n=0}^{\infty} b_{n}=\infty$ and by (22), we get $\lim \sup _{n \rightarrow \infty} c_{n}$ $\leq 0$. Consequently, applying Lemma 2.5 , to (31), we conclude that $x^{n} \rightarrow x^{*}$. Since $\| y_{n}$ - $x^{*}\|\leq\| x_{n}-x^{*} \|$, we have $y^{n} \rightarrow x^{*}$.

Corollary 3.2 Let $\left\{\alpha_{n}\right\},\left\{\beta_{n}\right\},\left\{\delta_{n}\right\}$ and $B$ be as in Theorem 3.1. Let $T$ a nonexpansive mapping of $C$ into $C$ such that $\mathcal{F}=\operatorname{Fix}(T) \cap \operatorname{VI}(C, B) \neq \emptyset$. Suppose $x_{0} \in H$ and $\left\{x_{n}\right\}$ and $\left\{y_{n}\right\}$ be generated by the iteration algorithm

$$
\left\{\begin{array}{l}
y_{n}=\beta_{n} x_{n}+\left(1-\beta_{n}\right) P_{C}\left(x_{n}-\delta_{n} B x_{n}\right), \\
x_{n+1}=\alpha_{n} \gamma f\left(x_{n}\right)+\left(I-\alpha_{n} \mu F\right) \frac{n}{n-1} \int_{0}^{\frac{n-1}{n}} T(t) y_{n} d t, \quad n \geq 0 .
\end{array}\right.
$$

Then $\left\{x_{n}\right\}$ and $\left\{y_{n}\right\}$ convergence strongly to $x^{*}$ which is the unique solution of the systems of variational inequalities: 


$$
\begin{cases}\left\langle(\mu F-\gamma f) x^{*}, x-x^{*}\right\rangle \geq 0, & \forall x \in \mathcal{F} \\ \left\langle B x^{*}, y-x^{*}\right\rangle \geq 0 & \forall y \in C\end{cases}
$$

Proof. Take $\lambda_{n}=\frac{n-1}{n}$, for $n \in \mathbb{N}$, we define $\mu_{n}(f)=\frac{1}{\lambda_{n}} \int_{0}^{\lambda_{n}} f(t) \mathrm{d} t$ for each $f \in C\left(\mathbb{R}_{+}\right)$, where $C\left(\mathbb{R}_{+}\right)$denotes the space of all real-valued bounded continuous functions on $R^{+}$ with supremum norm. Then, $\left\{\mu_{n}\right\}$ is regular sequence of means on $C\left(\mathbb{R}_{+}\right)$such that

$$
\left\|\mu_{n+1}-\mu_{n}\right\| \leq 2\left(1-\frac{\lambda_{n}}{\lambda_{n+1}}\right)
$$

for more details, see [21]. Further, for each $y \in C$, we have

$$
T_{\mu_{n}} y=\frac{1}{\lambda_{n}} \int_{0}^{\lambda_{n}} T(t) y \mathrm{~d} t .
$$

On the other hand

$$
\begin{aligned}
\sum_{n=1}^{\infty}\left\|\mu_{n+1}-\mu_{n}\right\| & \leq 2 \sum_{n=1}^{\infty}\left(\frac{\lambda_{n+1}-\lambda_{n}}{\lambda_{n+1}}\right) \\
& =2 \sum_{n=1}^{\infty}\left(\frac{\frac{n}{n+1}-\frac{n-1}{n}}{\frac{n}{n+1}}\right) \\
& =2 \sum_{n=1}^{\infty} \frac{1}{n^{2}}<\infty
\end{aligned}
$$

Now, apply Theorem 3.1 to conclude the result.

Corollary 3.3 Let $S, \phi, X,\left\{\mu_{n}\right\}, \mathcal{F},\left\{\alpha_{n}\right\},\left\{\beta_{n}\right\}$, and $\left\{\delta_{n}\right\}$ be as in Theorem 3.1. Let $A$ be a strongly positive bounded linear operator with coefficient $\bar{\gamma}>0, \zeta$ a number in $\left(0, \frac{\bar{\tau}}{\alpha}\right)$, where $\bar{\tau}=\bar{\mu}\left(\bar{\gamma}-\frac{\bar{\mu}\|A\|^{2}}{2}\right)$ and $0<\bar{\mu}<\frac{2 \bar{\gamma}}{\|A\|^{2}}$. If $\left\{x_{n}\right\}$ and $\left\{y_{n}\right\}$ are generated by $x_{0} \in C$ and

$$
\left\{\begin{array}{l}
y_{n}=\beta_{n} x_{n}+\left(1-\beta_{n}\right) P_{C}\left(x_{n}-\delta_{n} A x_{n}\right), \\
x_{n+1}=\alpha_{n} \gamma f\left(x_{n}\right)+\left(I-\alpha_{n} \bar{\mu} A\right) T_{\mu_{n}} y_{n}, \quad n \geq 0 .
\end{array}\right.
$$

Then, $\left\{x_{n}\right\}$ and $\left\{y_{n}\right\}$ converge strongly, as $n \rightarrow \infty$, to $x^{*} \in \mathcal{F}$, which is a unique solution of the variational inequalities:

$$
\begin{cases}\left\langle(\mu F-\gamma f) x^{*}, x-x^{*}\right\rangle \geq 0, & \forall x \in \mathcal{F}, \\ \left\langle A x^{*}, y-x^{*}\right\rangle \geq 0 & \forall y \in C .\end{cases}
$$

Proof. Because $A$ is strongly positive bounded linear operator on $H$ with coefficient $\bar{\gamma}$, we have

$$
\langle A x-A y, x-y\rangle \geq \bar{\gamma}\|x-y\|^{2} .
$$

Therefore, $A$ is $\bar{\gamma}$-strongly monotone.

On the other hand

$$
\|A x-A y\| \leq\|A\|\|x-y\| .
$$

Therefore,

$$
\frac{\bar{\gamma}}{\|A\|^{2}}\|A x-A y\|^{2} \leq\langle A x-A y, x-y\rangle .
$$


Hence, $A$ is $\frac{\bar{\gamma}}{\|A\|^{2}}$-inverse strongly monotone. Now apply Theorem 3.1 to conclude the result. $\square$

Corollary 3.4 Let $\left\{\alpha_{n}\right\},\left\{\beta_{n}\right\}$ and $B$ be as in Theorem 3.1. Let $u, x_{0} \in C$ and $\left\{x_{n}\right\}$ and $\left\{y_{n}\right\}$ be generated by the iterative algorithm

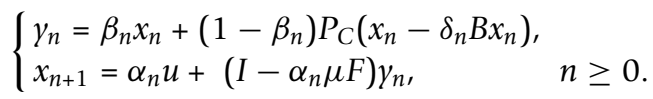

Then $\left\{x_{n}\right\}$ and $\left\{y_{n}\right\}$ convergence strongly to $x^{*}$ which is the unique solution of the systems of variational inequalities:

$$
\begin{cases}\left\langle(\mu F-\gamma f) x^{*}, x-x^{*}\right\rangle \geq 0, & \forall x \in \mathcal{F}, \\ \left\langle B x^{*}, y-x^{*}\right\rangle \geq 0 & \forall y \in C .\end{cases}
$$

Proof. It is sufficient to take $f=\frac{1}{\gamma} u$ and $\phi=\{I\}$ in Theorem 3.1.

\section{Acknowledgements}

The authors are extremely grateful to the reviewers for careful reading, valuable comment and suggestions that improved the content of this paper. This work is supported by University of Bonab under Research Projection 100-22.

\section{Authors' contributions}

The authors have equitably contributed in obtaining the new results presented in this article. All authors read and approved the final manuscript.

\section{Competing interests}

The authors declare that they have no competing interests.

Received: 25 November 2010 Accepted: 19 September 2011 Published: 19 September 2011

\section{References}

1. Yao, JC, Chadli, O: Pseudomonotone complementarity problems and variational inequalities, Handbook of generalized convexity and generalized monotonicity. Nonconvex Optim Appl.76, 501-555

2. Zeng, LC, Schaible, S, Yao, JC: Iterative algorithm for generalized set-valued strongly nonlinear mixed variational-like inequalities. J Optim Theory Appl. 124, 725-738 (2005). doi:10.1007/s10957-004-1182-z

3. Moudafi, A: Viscosity approximation methods for fixed-points problems. J Math Anal Appl. 241, 46-55 (2000). doi:10.1006/jmaa.1999.6615

4. Xu, HK: Viscosity approximation methods for nonexpansive mappings. J Math Anal Appl. 298, 279-291 (2004). doi:10.1016/j.jmaa.2004.04.059

5. Xu, HK: An iterative approach to quadratic optimization. J Optim Theory Appl. 116, 659-678 (2003). doi:10.1023/ A:1023073621589

6. Marino, G, Xu, HK: A general iterative method for nonexpansive mappings in Hilbert spaces. J Math Anal Appl. 318, 43-52 (2006). doi:10.1016/j.jmaa.2005.05.028

7. Liu, Y: A general iterative method for equilibrium problems and strict pseudo-contractions in Hilbert spaces. Nonlinear Anal. 71, 4852-4861 (2009). doi:10.1016/j.na.2009.03.060

8. Qin, X, Shang, M, Kang, SM: Strong convergence theorems of modified Mann iterative process for strict pseudocontractions in Hilbert spaces. Nonlinear Anal. 70, 1257-1264 (2009). doi:10.1016/j.na.2008.02.009

9. Yamada, I: The hybrid steepest descent method for the variational inequality problem over the intersection of fixed point sets of nonexpansive mappings, Inherently parallel algorithms in feasibility and optimization and their applications (Haifa, 2000). Stud Comput Math. 8, 473-504 (2001)

10. Tian, M: A general iterative algorithm for nonexpansive mappings in Hilbert spaces. Nonlinear Anal. 73, 689-694 (2010). doi:10.1016/j.na.2010.03.058

11. Atsushiba, S, Takahashi, W: Approximating common fixed points of non-expansive semigroups by the Mann iteration process. Proceedings of Workshop on Fixed Point Theory (Kazimierz Dolny, 1997). 51, 1-16 (1997)

12. Ceng, LC, Yao, JC: A relaxed extragradient-like method for a generalized mixed equilibrium problem, a general system of generalized equilibria and a fixed point problem. Nonlinear Anal. 72, 1922-1937 (2010). doi:10.1016/j.na.2009.09.033

13. Kim, KS: Convergence of a hybrid algorithm for a reversible semigroup of nonlinear operators in Banach spaces. Nonlinear Anal. 73, 3413-3419 (2010). doi:10.1016/j.na.2010.07.027

14. Lau, AT, Miyake, H, Takahashi, W: Approximation of fixed points for amenable semigroups of nonexpansive mappings in Banach spaces. Nonlinear Anal. 67, 1211-1225 (2007). doi:10.1016/j.na.2006.07.008

15. Lau, AT, Shioji, N, Takahashi, W: Existence of nonexpansive retractions for amenable semigroups of nonexpansive mappings and nonlinear ergodic theorems in Banach spaces. J Funct Anal. 161, 62-75 (1999). doi:10.1006/ jfan.1998.3352

16. Piri, H, Vaezi, H: Strong Convergence of a Generalized Iterative Method for Semigroups of Nonexpansive Mappings in Hilbert Spaces. Fixed Point Theory and Applications 2010, 16 (2010). Article ID 907275 
17. Agarwal, RP, O'Regan, D, Sahu, DR: Fixed point theory for Lipschitzian-type mappings with applications. In Topological Fixed Point Theory and Its Applications, vol. 6,Springer, New York (2009)

18. Jitpeera, T, Kumam, P: An extragradient type method for a system of equilibrium problems, variational inequality problems and fixed point of finitely many nonexpansive mappings. J Nonlinear Anal Optim. 1, 71-91 (2010)

19. Rockafellar, RT: On the maximality of sums of nonlinear monotone operator. Trans Amer Math Soc. 149, 75-88 (1970). doi:10.1090/50002-9947-1970-0282272-5

20. Bruck, RE: On the convex approximation property and the asymptotic behavior of nonlinear contractions in Banach spaces. Israel J Math. 38, 304-314 (1981). doi:10.1007/BF02762776

21. Eshita, K, Miyake, H, Takahashi, W: Strong convergence theorems for asymptotically nonexpansive mappings in general Banach spaces, Dynamics of Continuous. Discret Impuls Syst Ser A Math Anal. 13, 621-640 (2006)

doi:10.1186/1687-1812-2011-55

Cite this article as: Piri and Badali: Strong convergence theorem for amenable semigroups of nonexpansive mappings and variational inequalities. Fixed Point Theory and Applications 2011 2011:55.

\section{Submit your manuscript to a SpringerOpen ${ }^{\circ}$} journal and benefit from:

$\rightarrow$ Convenient online submission

- Rigorous peer review

- Immediate publication on acceptance

- Open access: articles freely available online

- High visibility within the field

- Retaining the copyright to your article

Submit your next manuscript at $\boldsymbol{\wedge}$ springeropen.com 